$B \times a y+$ and

Lanre 



\title{
Die Brennhaare der Euphorbiaceen-Gattungen Dalechampia und Tragia
}

\author{
von
}

F. Knoll.

Aus dem Botanischen Institut der Universität Graz.

(Mit 2 Tafeln.)

(Vorgelegt in der Sitzung am 16. Februar 1905.)

Schon seit langer Zeit ist es bekannt, daß die jungen Früchte der in Brasilien einheimischen Acalyphee Tragia volubilis Mich x. mit einem dichten Überzug eigentümlich gebauter Brennhaare versehen sind. Crüger erwähnt 185う: „Es sind sehr schöne prismatische Brennhaare, bestehend aus vier bis fünf langen Zellen, von denen eine die Mitte einnimmt und welche von einer zugespitzten Zelle gekrönt sind. Diese Zelle ist an ihrer Basis porös. Diejenige der langen Zellen, welche von den anderen umgeben ist, bleibt fast ganz unverdickt und ich fand sie erst bei der Zerlegung der Haare bei der Mazeration. Die darum herumstehenden Zellen werden ziemlich stark verdickt. «

Von der gleichen Pflanze sagt Kohl: »Jedes ihrer Haare besteht aus drei langen, nebeneinander liegenden, dickwandigen Zellen und einer diesen aufsitzenden spitzen, zartwandigen Endzelle, welche einen oder bisweilen zwei große Spießkristalle von oxalsaurem Kalk einschließt."A. Weiß wiederholt fast wörtlich die von Cr üger gemachten Angaben. Eine Notiz, welche nach Rittershausen in Stahls Arbeit »Pflanzen und Schnecken « vorkommen soll, konnte ich nicht auffinden. Im Jahre 1892 hat Rittershausen diese Brennhaare genauer untersucht und für viele Arten der Acalypheen nachgewiesen. Er unterscheidet "große» und »kleine Brennhaare.

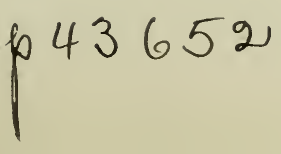


des lebenden Protoplasten, nur sehr wenig. Weit besser lassen sich die Verhältnisse untersuchen, wenn das Material nach Fixierung mit Alkohol 24 Stunden in Eau de Javelle gelegt und nach dem Auswaschen mit Wasser etwa gleich lange Zeit mit zehnprozentiger Essigsäure nachbehandelt wird. Die meisten Details lassen sich an dem so vorbereiteten Materiale durch verschieden hohe Einstellung des Mikroskops ermitteln; für die feineren Untersuchungen ist die Benützung von Mikrotomschnitten erforderlich.

Nach den von mir gefundenen Tatsachen kann der Bau eines normal entwickelten ausgewachsenen Brennhaares in Kürze folgendermaßen zusammengefaßt werden. Du rch e inen Sockel von drei bis fünf hoch emporgehobenen Epidermiszellen (ich nenne sie »Seitenzellen ", »Außenzellen «) zieht sich, etwas unter dem Niveau der Epidermisinnenwand beginnend, eine langgestreckte Zelle ("Zentralzelle"), welche mit ihrem zugespitzten Ende, das einen Kristall aus oxalsaurem Kalk enthält, weit über das Ende des Sockels emporragt. Fig. 5 der beiliegenden Tafel I gibt ein klares Bild dieser Verhältnisse bei der Gattung Dalechampia. Die Brennhaare von Tragia volubilis Michx., welche mir nebst anderen Tragia-Arten in Herbarexemplaren vorlag, zeigen in den vorerwähnten Details das gleiche Verhalten wie die von Dalechampia. Der Fehler aller bisherigen Beobachtungen besteht also vor allem darin, daß man von einer birnförmigen Zelle sprach, welche der mittleren Zelle des Sockels aufsitzen sollte, somit in der Annahme einer (in Wirklichkeit nicht vorhandenen) Scheidewand zwischen dem oberen und unteren Teil der Zentralzelle. Schon aus Fig. 10 und 11 der Arbeit Rittershausens ergibt sich, daß die beiden darauffolgenden Figuren 12 und 13 unrichtig sein müssen, ivenn, wie schon dieser Autor selbst vermutet, die "großen " und »kleinen « Brennhaare nur als Entwicklungsstadien aufzufassen sind.

Die Zentralzelle ist die eigentliche Brennhaarzelle. Die Außenzellen bilden den dazugehörigen Hilfsapparat. Die Zentralzelle steckt mit dem etwas verdickten Fußteil zwischen dem subepidermalen Zellgewebe und ist innerhalb des Sockels 
Die von den vorigen Autoren beschriebenen Brennhare gehören zu den "großen « Brennhaaren. Die »kleinen ", »einfachen "Brennhaare bestehen "aus einer einzigen haarförmigen Zelle, in deren Spitze, an Zellstoffbalken befestigt, ein pfriemenartiger, nach oben nadelscharf zulaufender Kristall aus oxalsaurem Kalke hängt. An seinem unteren Ende besitzt er zwei bis drei kleine abyestumpfte Zacken. Sehr oft ist dieses einzellige Brennhaar an seinem unterem Teile bauchig erweitert." Rittershausen erwähnt solche Brennhaare für die Gattungen Tragia, Cnesmone, Leptorhachis und Dalechampia und benützt das Vorkommen dieser Haare zur Sicherung der systematischen Stellung der letzterwähnten Gattung. Zuletzt wurden diese Brennhaare von Solereder untersucht, der zu den gleichen Resultaten kam wie Rittershausen. Die von letzterem gebotenen unklaren Abbildungen hat Solereder durch deutlichere ersetzt.

Da diese Brennhaare einen so auffallenden Bau besitzen und die schon vorhandenen Angaben ziemlich unvollkommen sind, habe ich dieselben einer genauen Untersuchung unterzogen. Die interessanten Ergebnisse derselben will ich der größeren Übersichtlichkeit wegen in zwei gesonderten Teilen darlegen.

\section{Bau und Funktion der Acalypheen-Brennhaare.}

(Hiezu Tafel I.)

Meine über diesen Gegenstand gemachten Beobachtungen und Untersuchungen beziehen sich vor allem auf die im Gewächshause des hiesigen botanischen Gartens kultivierte Dalechampia Roezliana a rosea Müll. Arg., welche das ganze Jahr hindurch reichlich blüht und an den zarten, rosenrot gefärbten Hochblättern jene erwähnten Brennhaare stets in großer Anzahl hervorbringt.

Schon bei schwächerer Vergrößerung sieht man die glashellen, mit stark reflektierenden glänzenden Außenwänden versehenen Haare längs des Blattrandes und an der Unterseite der Haupt- und Nebennerven. Die vollkommen entwickelten frischen Brennhaare zeigen uns, abgesehen von der Beschaffenheit 
so außerordentlich dünnwandig, daß sie hier in den meisten Fällen ohne eine entsprechende Präparation überhaupt nicht sichtbar ist. Darauf hat schon Crüg er hingewiesen. Dadurch ist auch erklärlich, daß Kohl nur von drei Sockelzellen spricht - die zentral gelegene Zelle ist ihm jedenfalls entgangen. Derjenige Teil der Zentralzelle, welcher über die Seitenzellen hinausragt, zeigt unten eine sehr dicke Außenwand, welche dann rasch an Dicke abnimmt, in eine längere, sehr dünne Partie übergeht und an der Spitze des Haares mit einer kappenartigen Verdickung endigt. Die Beschaffenheit dieser kappenartigen Endverdickung wird später noch genauer dargelegt werden.

An jener Stelle, wo die Zentralzelle den Sockel verläßt, zeigt sich, an Zellulosebalken aufgehangen, eine Kristalldruse, deren in der Richtung der Haarspitze gelegene Kristall auf Kosten der übrigen Kristallindividuen außerordentlich bevorzugt ist. Während der das freie Ende der̃ Zentralzelle durchziehende Kristall der Druse eine für die Stichfunktion ganz besonders günstige Beschaffenheit hat, sind die übrigen Kristalle meist nur als kleine Ecken oder Hervorragungen am unteren Ende des Spießkristalls ausgebildet (Fig. 15 und 17). Mitunter findet sich auch in entgegengesetzter Richtung ein mehr oder weniges ausgebildeter, spießförmiger Kristall entwickelt (Fig. 16). Der Hauptkristall zeigt an dem nach außen gewendeten Ende eine unter spitzem Winkel (zur Längsachse des Kristalls) gelegene Fläche (Fig. 17e). Die sehr großen Brennhaare von Tragia bicolor Miq. (=Tragia Miqueliana Mülll. Arg.) aus Ostindien lassen die Beschaffenheit des Kristalls besonders gut erkennen. Bei Tragia zeigt sich am Kristall außerdem eine unter sehr spitzem Winkel verlaufende (rinnenartige?) Seitenfläche (Fig. $17 \mathrm{~s}$ ), welche auch bei Dalechampia meist vorhanden ist, aber wegen der Kleinheit der Kristalle bei dieser Gattung wenig auffällt.

Die ganze Kristalldruse ist von einer Zellulosehülle eingeschlossen, welche jedoch nicht überall die gleiche Dicke aufweist. Besonders mächtig ist sie dort, wo die Kristalldruse durch Zellulosebalken in der Außenwand der Zentralzelle verankert ist. Diese Verankerung erstreckt sich bei Dalechampia 
auf die untere Hälfte, bei Tragia auf das untere Drittel des freien Endes der Zentralzelle. Der mittlere Teil des Spießkristalls wird von einer sehr dünnen, eng anliegenden Zelluloseschichte umhüllt, welche erst nach der Auflösung der Druse durch Salzsäure sichtbar gemacht werden kann (Fig. 5, 6 und 10). Bei Tragia Miqueliana Mü1l. ist die Verankerung entsprechend der bedeutenden Größe der Brenıhaare eine überaus kräftige. Kristallhülle und Zellwand sind durch dicke, deutlich geschichtete Balken verbunden, welche öfters untereinander zu massiven Platten verschmelzen (Fig. 10, 11, 13). In manchen Fällen (Fig. 12) beobachtete ich solche Platten von besonderer Größe, welche dann nur einige wenige kleine Löcher aufzuweisen hatten. Vielleicht wurden durch ähnliche Bildungen die bisherigen Beobachter zur Annahme von 'Tüpfeln in der von ihnen gedachten unteren Brennhaarwand gebracht. Oft sieht man in den Zellulosebalken ein feines Lumen (?), das sich in der Querschnittsansicht als Punkt darstellt. Jedenfalls hängt diese Bildung mit der Entstehungsweise der Balken an oder in Plasmafäden zusammen.

Die Spitze des Spießkristalls steckt in einer etwas dickeren Hülle von Zellulose, welche ohne deutliche Grenze in die kappenförmige Verdickung der Brennhaarspitze übergeht.

Die mit mäßig verdickten Außenwänden versehenen drei bis fünf Seitenzellen fixieren die Zentralzelle in der für die Brennhaarfunktion günstigsten Stellung. So stehen die Brennhaare an den Hochblättern von Dalechampia stets etwas schief von der Epidermis ab (Fig. 5), so daß sie nach außen gegen die Spitze des Blattes zu gerichtet sind.

Die Cuticula, welche das ganze Brennhaar überzieht, ist bei Dalechampia vollkommen glatt, während sie an den Seitenzellen älterer Brennhaare von Tragia kurze strichförmige Skulpturen aufweist (Fig. 13).

Im lebenden Zustande enthalten die Außenzellen der Dalechampia-Brennhaare stets sehr viel Protoplasma. Doch fand ich den Plasmareichtum bei den von mir untersuchten Brennhaaren nicht überall gleich groß. Der Zellsaftraum ist oft auf eine oder mehrere Vakuolen reduziert, oft aber sehr groß und von körnchenreichen Plasmasträngen durchzogen. Die Zellkerne der 
Seitenzellen sind häufig schon an dem lebenden Brennhaar zu erkennen; sie haben die Größe der in den Epidermiszellen vorhandenen Kerne und liegen meist der dünnen Innenwand der Außenzelle an. Der Zellkern der Zentralzelle ist größer als derjenige der Seitenzellen und liegt in ausgewachsenen Brennhaaren im oberen Teil in der Nähe der Kristalldruse (Fig. 9).

Die Zentralzelle der Dalechampia-Brennhaare enthält ebenfalls reichlich Protoplasma und, was sehr wichtig ist, große Mengen von Eiweißstoffen, welche im Zellsaft gelöst sind. Ameisensäure ist in der Zentralzelle (nach Rittershausen) nicht vorhanden. Jedenfalls deutet der große Eiweißgehalt der Zentralzelle auf eine Übereinstimmung mit den von Haberlandt untersuchten Brennhaaren und läßt vermuten, daß es sich wohl auch hier um die Absonderung eines fermentoder enzymartigen Giftes handelt. Die Seitenzellen sind frei von nachweisbaren Eiweißmengen. Bei längerem Liegen in absolutem Alkohol oder beim Kochen in Wasser bleiben die Seitenzellen vollkommen klar, während sich die Zentralzelle mit einem dichten undurchsichtigen Coagulum angefüllt hat, welches alle für Eiweiß charakteristischen Reaktionen zeigt. Außerdem enthalten die Zentralzellen bei Dalechampia meist eine Anzahl Stärkekörner, während bei Tragia volubilis Michx. die Seitenzellen sehr viel Stärke aufweisen. Bei letzterer wird die Stärke vielleicht zum größeren Teil an Ort und Stelle gebildet, da Crüger in den lebenden Brennhaaren derselben Pflanze »viele grobe, weiße und grünliche Körner « im Plasmastrome sich bewegen sah. Wahrscheinlich wird die in den ausgewachsenen Brennhaaren dieser Pflanze vorhandene Stärke bei der Giftbereitung aufgebraucht.

Ich habe die in Rede stehenden Organe von Dalechampia als »Brennhaare " bezeichnet, obwohl sich niemand mit ihnen zu brennen vermochte. Ritte rshausen konnte sich an den in München kultivierten Exemplaren von Dalechampia nicht verletzen, auch konnte ich selbst an den hier vorhandenen lebenden Exemplaren nicht einmal bei der Berührung mit der sonst so empfindlichen Zungenspitze eine Schmerzempfindung wahrnehmen. Rittershausen meint, daß es sich hier vielleicht um eine Kulturerscheinung handelt, so daß bei den in unseren 
Gewächshäusern kultivierten Dalechampia-Arten überhaupt kein Gift ausgebildet werde; ich halte es aber für wahrscheinlicher, daß die Brennhaare dieser Pflanzengattung für den Menschen überhaupt unschädlich sind. Gegen welche Feinde sich aber die Dalechampia-Brennhaare als nützlich erweisen, ließe sich nur in der Heimat der Pflanze, in Mexiko, genau feststellen. Soviel ist aber sicher, daß die ganz gleich gebauten Haare der Gattung Tragia auch den Menschen verletzen können. Müller Arg., welcher die Acalypheen in De Candolles Prodromus bearbeitete, sagt daselbst, die Tragia-Arten seien ».. frutices vel suffrutices vel herbae .... saepissime urticarum more pilis plus minusve vehementer urentibus vestitae «.

Frisches Material von Tragia ist leider nicht zu erhalten; ich mußte also trachten, mir am Herbarmateriale eine klare und möglichst sichere Vorstellung von der Funktion dieser Brennhaare $z u$ verschaffen. Ich ging dabei von dem Gedanken aus, daß Brennhaare, die ihren Zweck erfüllen sollen, ihr Gift in zweckmäßiger Weise in die vorerst geschaffene Wunde entleeren müssen. Wenn man z. B. an den bereits geöffneten Brennhaaren von Urtica die verkieselte Spitze stets schief abgebrochen findet, so ist das eine Eigentümlichkeit, welche durch die anatomische Beschaffenheit derselben $\mathrm{zu}$ stande kommt und dem Zweckmässigkeitsprinzip vollkommen entspricht. Das durch Verkieselung steife Ende dringt leicht in die Haut ein, die Spitze bricht an der präformierten Stelle ab und das Gift fließt durch die schiefe Ausflußöffnung beim Zurückziehen des Haares in genügender Menge in die Wunde. Daß im Brennhaare der Acalypheen der Spießkristall ein überaus günstiges Werkzeug darstellt, um eine Wunde zu schaffen, geht aus Fig. 17 deutlich genug hervor. Ein Abbrechen der Brennhaarspitze zur Schaffung einer zweckmäßig gelegenen Ausflußöffnung ist schon deshalb ausgeschlossen, weil das unverdickte Ende der Zentralzelle eine überaus elastische Beschaffenheit der Wand besitzt. Davon kann man sich leicht überzeugen, wenn man an die Spitze eines solchen Brennhaares, das sich gerade im Gesichtsfeld des Mikroskops befindet, von der Seite her, also normal auf die Längsachse des Haares, mit einem Glasfaden stark genug anstößt. Wenn durch einen stärkeren Stoß der 
Kristall entzweigebrochen ist, läßt sich dasjenige Ende des Haares, welches die Kristallspitze enthält, rechtwinkelig zur Seite biegen, um nach dem Zurückziehen des Glasfadens sofort in die ursprünglich gestreckte Lage zurückzukehren. Die Membran zeigt an der umgebogenen Stelle dann nur selten Spuren der vorgenommenen Knickung. Wenn der Kristall hier tatsächlich als Stichwaffe dient, würde eine feste, spröde Beschaffenheit der Haarspitze ein Durchbohren der Membran durch die Kristallspitze erschweren oder ganz verhindern. Ich habe früher erwähnt, daß die Brennhaarspitze eine starke kappenförmige Verdickung aufweist, welche mit dem oberen Teil der Kristallhülle verbunden ist. Diese Verdickung müßte, wenn an ihr nicht ganz besondere Einrichtungen getroffen wären, der hindurchdringenden Kristallspitze einen ziemlich bedeutenden Widerstand entgegensetzen. Um uns über diese Einrichtungen $\mathrm{zu}$ orientieren, wählen wir wieder am besten die großen Brennhaare von Tragia Miqueliana Mü1l. An allen unverletzten Brennhaaren werden wir sofort sehen, daß die äußerste Kristallspitze bis nahe an die Cuticula reicht (Fig. 14). Vollkommenen Aufschluß gewähren jene Brennhaare, welche den Kristall so orientiert zeigen, daß dessen Breitseite dem Beschauer zugekehrt ist (Fig. 14b). Da zeigt sich denn, daß die ganze schräge Endfläche fast unmittelbar unter der Cuticula zu liegen kommt. Hier haben wir die für das Hindurchdringen des Kristalls präformierte Stelle. - Mit seinem breiten unteren Ende steckt der Spießkristall unbeweglich in der festen Zelluloseverankerung, mit seinem oberen dünneren Ende in der Zellulosekappe der Brennhaarspitze. Daraus geht hervor, daß der Kristall nur dann die Brennhaarspitze durchbohren kann, wenn ein Stoß annähernd in der Richtung der Brennhaarachse das Ende der Zentralzelle trifft. Die Zellwand wird an der verdünnten Stelle gesprengt, die Kristallhülle an der zarten, in Fig. $14 a$ mit * bezeichneten Stelle zerrissen und das ganze dünnwandige Endstück der Kristailzelle kann nun unter der Führung der durchbohrten Zellulosekappe am entblößten Kristall zurückgeschoben werden (Fig. 18 und 19). Wenn der Turgor der Brennhaarzellen sich an der Entleerung des Giftes auch gar nicht beteiligt, so genügt die durch das Zusammenschieben 
der Membran des Zentralzellendes bewirkte Volumsverminderung, um eine wirksame Menge der giftigen Substanz in die Wunde ausfließen zu lassen. Die durch diesen Vorgang bloßgelegte schiefe Endfläche des Spießkristalls bewirkt nun dasselbe wie die schief abgebrochene Spitze der Urtica-Brennhaare.

Mit der einmaligen Funktion ist der Zweck des Brennhaares erfüllt; eine Regeneration findet nicht mehr statt. An den Brennhaaren von Tragia volubilis Michx. bleibt das Ende der Zentralzelle oft am Kristall zurückgeschoben, wo es jedenfalls antrocknet. Bei Tragia Miqueliana Mü1l. Arg. sah ich nur sehr selten Stadien, wie ich sie für die vorerwähnte Art in Fig. 18 und 19 abgebildet habe, dagegen die Zellulosekappe in sehr vielen Fällen durchbohrt. Es scheint, daß sich in diesen Fällen die Membran infolge ihrer Elastizität nachträglich in die ursprüngliche Lage zurückbegeben hat.

Kohl sagt über die Funktion der Brennhaare von Tragia volubilis: „Unmittelbar an die Rhaphiden schließen sich in ihrer Wirkung als Schutzmittel die großen Kalkoxalatkristalle an, welche in den Brennhaaren einiger Pflanzen als Stichwaffen funktionieren...... Bei jeder unsanften Berührung der Haarspitze hohrt sich der Kristall durch die dünne Membran der Endzelle hindurch in die Haut ein und verursacht, in der Wunde stückweise stecken bleibend, ein unangenehmes Jucken. Noch ist es nicht bekannt, ob gleichzeitig ein flüssiges Gift vom Haare entleert wird. "Ich weiß nicht, ob Kohl diese Wahrnehmung an sich selbst gemacht hat oder ob diese Äußerung nur eine Darlegung dafür bietet, wie sich Kohl die Funktion der Brennhaare vorstellt. Ich halte es für möglich, daß mitunter ein Stück des Kristalls in der Wunde stecken bleiben kann - ich glaube aber, daß das nur zufällig geschieht. Denn an den von mir untersuchten Brennhaaren der Tragia volubilis fand ich den Kristall fast immer unversehrt, trotzdem die meisten derselben geöffnet waren und die Endmembran der Zentralzelle oft bis zur Verankerung zurückgeschoben war. Wenn der Kristall abgebrochen war, fand ich stets die Zellhaut an der betreffenden Stelle durch- oder ganz weggerissen, was natürlich, da es sich um Herbarmaterial handelte, im 
getrockneten Zustand leicht geschehen sein konnte. - Jedenfalls bringen diese Brennhaare ein weiteres Argument für die von L. Lew in angenommene Bedeutung der Rhaphiden; denn auch hier schafft der spießförmige Kristall eine Wunde für ein in der Zelle vorhandenes Gift. Im übrigen unterscheiden sich die Rhaphiden wesentlich dadurch von dem Kristallapparat der Brennhaare, daß die Rhaphiden von allem Anfang an als vollkommen ausgebildete, in Bündeln nebeneinander liegende Einzelkristalle entstehen, während der Spießkristall einer umgebildeten Kristalldruse angehört.

Ich muß hier noch einmal darauf zurückkommen, daß die Brennhaare von Dalechampia Roezliana Müll. Arg. nach meiner Ansicht für den Menschen unschädlich sind. Wenn diese Brennhaare eine fühlbare Wirkung hervorbringen sollen, muß der Spießkristall zuerst eine ausreichend große Verwundung zu stande bringen können. Nun beträgt aber die Gesamtlänge des Kristalls hier im besten Falle nur $50 \mu$ ! Da das untere Ende noch in der dicken Zellulosehülle der Verankerung steckt, kann der Kristall, wenn die Zellwand möglichst weit zurückgeschoben wird, höchstens $20 \mu$ tief in die Haut eindringen. Ferner dürfte der ganze Hilfsapparat zur Übertragung des Giftes bei Dalechampia viel zu schwach gebaut sein, um den ziemlich großen Widerstand zu überwinden, welchen die menschliche Haut der Verwundung entgegensetzt. Denn wenn man an einem lebenden Hochblatt von Dalechampia einige Male auf der Unterseite des Blattes mit dem Finger gegen die Basis streift und dann die dadurch vielfach deformierten Brennhaare untersucht, so findet man in vielen Fällen den Kristall entzweigebrochen in der Zentralzelle, oft aber auch unverletzt in den zwischen den Seitenzellen befindlichen Teil der Kristallzelle hinabgesunken. Leicht begreiflich erscheint es dagegen, daß sich mit den sehr spitzen, oft bis $170 \mu$ langen Kristallen von Tragia Miqueliana Müll. Arg. auch der Mensch hinreichend verletzen kann, zumal da der Spießkristall außerordentlich gut verankert ist.

Wenn es auch, wie Habe rlandt sagt, »in erster Linie auf den spezifischen Charakter und nicht auf die Quantität des entleerten Giftes ankommt «, so möchte ich doch der Vollständigkeit 
wegen einige Zahlen anführen, welche uns eine Vorstellung von den Di mensionen dieser Brennhaare ermöglichen.

Die Größenverhältnisse sind ohneweiters aus folgender Tabelle ersichtlich; jede Zeile repräsentiert das Ergebnis der Messungen an einem einzelnen Brennhaar.

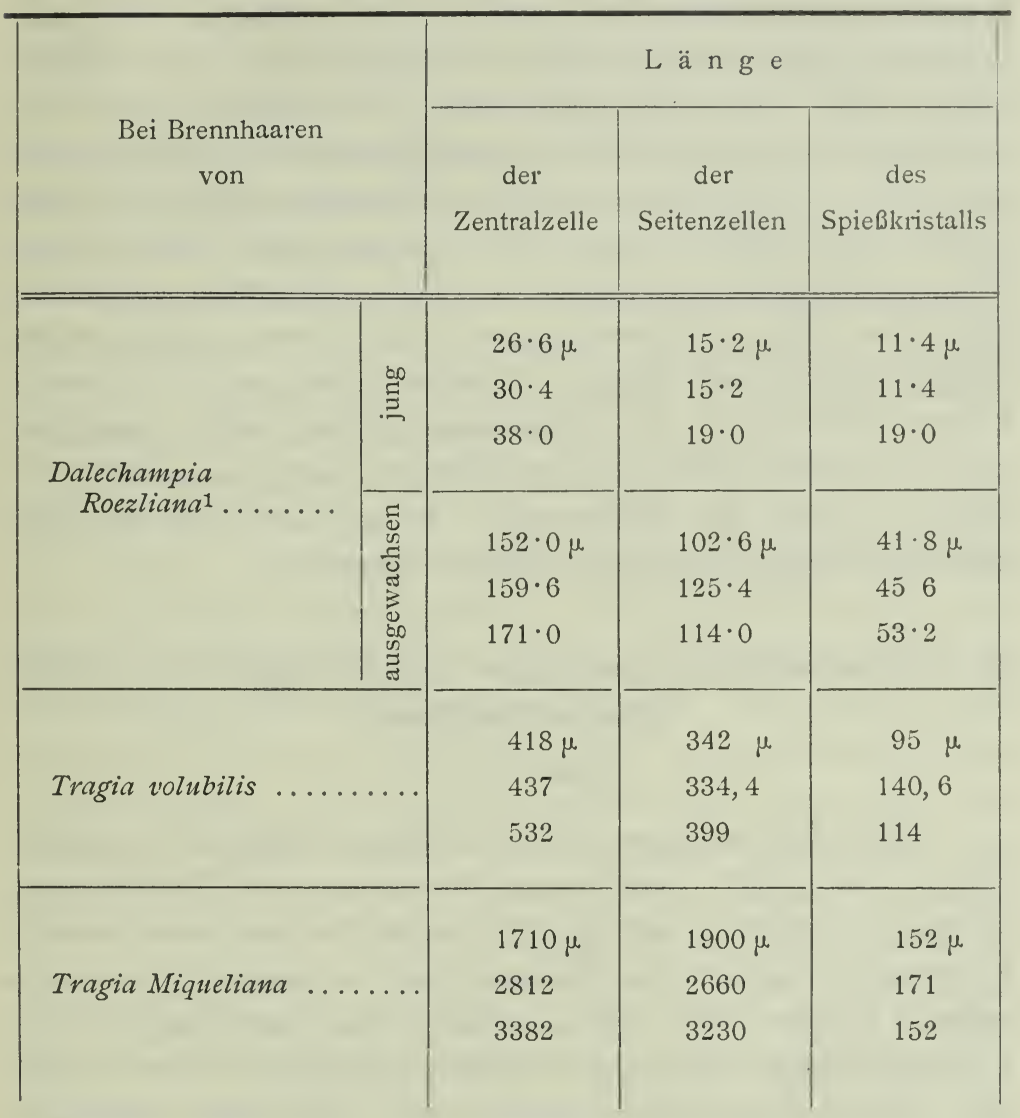

Für eine mittelgroße Zentralzelle von Dalechampia Roezliana berechnete ich ein Volumen von $0.000009234 \mathrm{~mm}^{3}$, für eine solche von Tragia Miqueliana ein Volumen von

1 Zum Vergleich sei erwähnt, daß die Epidermiszellen bei Dalechampıa eine Höhe von $15 \cdot 2 \mu$ besitzen. 
$0.00023 \mathrm{~mm}^{3}$; bei den Brennhaaren von Urtica beträgt der Gesamtinhalt der Brennhaarzelle im Mittel 0.007 bis $0.008 \mathrm{~mm}^{3}$ (nach $\mathrm{Haberlandt}$ ).

Die im vorigen geschilderten Brennhaare finden sich bei den von mir untersuchten Arten von Tragia und Dalechampia besonders zahlreich in der Blütenregion und an jungen Laubsprossen, wo sie stets in der Gesellschaft mehr oder weniger langer unverzweigter einzelliger Haare vorkommen. Daraus geht hervor, daß jene Organe zum Schutze der sich entwickelnden Laubblätter und Blütenteile, ganz besonders aber der heranwachsenden Früchte dienen. Die zerteilteńn Kelchblätter an den sich entwickelnden Früchten von Tragia Miqueliana sind mit einem überaus dichten, verderbendrohenden Pelz von $3 \mathrm{~mm}$ langen Brennhaaren versehen; bei Tragia volubilis ist die junge Frucht außen ganz von Brennhaaren überzogen. Die Brennhaare finden sich außerdem noch an anderen Teilen dieser Pflanzen, wenn auch sehr verstreut, so daß die Blütenregion dieser Gewächse die bestbewehrte Region der ganzen Pflanze darstellt.

\section{Die Entwicklungsgeschichte und Phylogenie der Acaly- pheen-Brennhaare.}

(Hiezu Tafel II.)

Betrachtet man den auf Taf. I, Fig. 5, dargestellten Längsschnitt durch ein erwachsenes Brennhaar von Dalechampia, so muß es auffallen, daß die Zentralzelle mit ihrem unteren Ende so tief unter das Niveau der inneren Epidermiswände hinabreicht. Diese Tatsache kann auf zweifache Art zu stande gekommen sein. Entweder entsteht die Zentralzelle aus einer Epidermiszelle, welche sich nach unten verlängert und zwischen die Zellen des unter der Epidermis gelegenen Gewebes hineinwächst, oder die Zentralzelle ist subepidermalen Ursprungs, durchdringt die Epidermis und wächst, gestützt von den mitwachsenden benachbarten Epidermiszellen (»Seitenzellen«) weit über die Außenfläche der Epidermis hinaus. Daß subepidermal gelegene Zellen tatsächlich imstande sind, durch gleitendes Wachstum bis in die 
Epidermis vorzudringen, hat W. Rothert für die Kristallzellen der Pontederiaceen und H. v. Guttenbèrg für Citrus nachgewiesen.

Ich habe schon früher mitgeteilt, daß sich die Zentralzellen der jungen Dalechampia-Brennhaare durch einen bedeutenden. Gehalt an Stärke auszeichnen. Wir wollen diesen Umstand benützen, um die ersten Anfänge der Brennhaarbildung ausfindig zu machen. Junge Hochblätter von Dalechampia werden zu diesem Zwecke in der im ersten Abschnitt dieser Arbeit angegebenen Weise mit Eau de Javelle und einer'zehnprozentigen Essigsäure behandelt und auf einige Zeit in Jodvasser gelegt und in Jodglyzerin untersucht. Die Stärkekörner sind durch Eau de Javelle nicht gelöst worden und haben sich durch Jod schwarzblau gefärbt; der sonst sehr störende Protoplast dagegen ist ganz verschwunden und die Zellen sind dadurch vollkommen durchsichtig geworden.

Wir untersuchen nun an den so präparierten jungen Hochblättern die Unterseite der Blattbasis und der Nerven, sowie den Blattrand. Bei entsprechender Einstellung finden wir bald subepidermal gelegene Zellen, welche sich von den benachbarten durch etwas geringere Größe und besonders durch den vorerwähnten Stärkereichtum auszeichnen. Eine solche Zelle zeigt uns Fig. 10 auf Taf. II. Sie liegt an der Grenze dreier Epidermiszellen, die Epidermis darüber zeigt noch nichts Auffallendes. In Fig. 9 dagegen hat sich die stärkeführende Zelle keilförmig nach oben verschmälert und ist gerade im Begriff, die beiden ober ihr liegenden Epidermiszellen auseinander zu drängen. Bald treten die Epidermiszellen etwas auseinander und die junge Zentralzelle tritt aus der Tiefe hervor. Sie ist entweder »Zweischneidig « (Fig. 9, 11, 12) oder »dreischneidig « (Fig. $8 a \alpha, 10,13$ ), je nachdem das Hindurchdringen an der Grenze zweier oder dreier Epidermiszellen erfolgte. In manchen Fällen ist die Zentralzelle gezwungen, in schiefer Richtung empor $z u$ wachsen; es bildet sich dann ein Buckel an der darüber liegenden Epidermiszelle (Fig. $8 a \beta, 8$ b). Wenn die Zentralzelle nach Art von Fig. 10 angelegt wird, dann ist ein Durchdringen leicht möglich; wenn aber die Anlage mitten unter eine größere Epidermiszelle zu liegen kommt, ist 
eine Weiterentwicklung entweder ausgeschlossen oder es tritt im richtigen Zeitpunkte eine Teilung der betreffenden Epidermiszelle ein. Solche Teilungen scheinen in der Tat sehr oft vorzukommen. Ein gutes Beispiel hiefür bietet Fig. 9, wo die Kontur der ursprünglichen Epidermiszelle noch deutlich sichtbar ist. Sehr auffallend ist es auch, daß die (im Verhältnis zur Größe der Epidermiszellen) ziemlich kleinen Zentralzellenanlagen fast immer wie in Fig. 10 entstehen. Daß nicht jede beliebige, für ein Durchdringen der Epidermis günstig gelegene subepidermale Zelle zur Zentralzelle auswachsen kann, zeigt schon der Umstand, daß die dazu befähigten Zellen sehr plasma- und stärkereich sind und in kleinen Dimensionen verharren, während ihre Nachbarinnen oft schon sehr stark herangewachsen sind. Sie scheinen also gleichsam ihre ganze Entwicklungs- und Wachstumsfähigkeit für jenen Zeitpunkt zu sparen, der ihnen infolge günstiger Zellteilungen ein Durchdringen der Epidermis ermöglicht. Vielleicht werden die Epidermiszellen durch die darunter liegenden Brennhaaranlagen zugleich in irgend welcher Weise veranlaßt, sich entsprechend zu teilen. - Fig. 13 zeigt (etwas schief von der Seite gesehen) ein an einem Blattnerv entstehendes Brennhaar, das in seinem oberen Teile bereits eine kleine Kristalldruse ausgebildet hat. Die Kristallindividuen sind aber noch vollkommen gleichartig; eine Andeutung des Spießkristalls ist noch nicht vorhanden. In diesem Stadium dürften bereits Zellulosebalken entwickelt sein, welche die Druse in ihrer Lage im oberen Teile der ganzen Zentralzelle festhalten.

Wir wollen nun die in der Oberflächenansicht beobachteten Entwicklungsstadien auch am Blattquerschnitt betrachten. Die Figuren 1 bis 7 sind nach Mikrotomschnitten gezeichnet worden und sie geben uns in ihrer Reihenfolge von 1 bis 4 ein vollkommen klares Bild der sich entwickelnden Brennhaaranlage. Fig. 1 zeigt uns eine Brennhaaranlage von einem etwa $1 \mathrm{~mm}$ langen Hochblatt von Dalechampia. Das Präparat ist mit Hämatoxylin gefärbt und zeigt vor allem den Plasma- und Stärkereichtum der jungen Zentralzelle. Auch sieht man ihre geringe Größe sehr deutlich an den benachbarten subepidermalen Zellen. Nach oben zu ist sie keilförmig zwischen zwei 
Epidermiszellen eingedrungen und hat dieselben im unteren Teile schon weit auseinandergedrängt. Im oberen Teil der Epidermiszellen ist die Mittellamelle noch nicht gespalten. Fig. 2 zeigt uns eine Anlage, in welcher die künftige Zentralzelle ihren ursprünglichen Platz zur Hälfte verlassen und noch ein Drittel der Epidermisdicke zu überwinden hat. In Fig. 3 - der Schnitt ist etwas seitlich geführt - hat die junge Zentralzelle die Außenfläche der Epidermis erreicht und die späteren Seitenzellen bereits mit emporgehoben. Fig. 4 zeigt $z w e i$ junge Brennhaare; bei $\beta$, das ebenfalls einen etwas seitlich geführten Schnitt darstellt, ist bereits der Spießkristall ausgebildet - der untere 'Teil der Kristalldruse ist beim Schneiden des Objektes weggebrochen. Die benachbarten Epidermiszellen werden zu Seitenzellen; doch treten unterdessen noch Teilungen in den ersteren auf, so daß aus den ursprünglich »Zweischneidigen « und »dreischneidigen « Zentralzellen meist vierseitige Brennhaare gebildet werden. Oft ist der Kristallapparat einer Zentralzelle schon vollständig ausgebildet, die Seitenzellen sind jedoch noch nicht emporgehoben (Fig. 5 und 7); das sind die »kleinen Brennhaare « im Sinne Rittershausens. Meist tritt jedoch das Emporheben der Seitenzellen gleich nach dem Durchdringen der Epidermis ein (Fig. 4 und 6) und dann wachsen Seitenzelle und Zentralzelle gemeinsam in die Länge. Von dem Wachstum und der Gestalt der Seitenzellen wird nun die Richtung der Brennhaarspitze und der Habitus des ganzen Haares bestimmt. Wachsen die Seitenzellen gerade in die Länge, dann entstehen Brennhaare wie Fig. 1, Taf. I; wachsen sie aber spiralig, dann entstehen gedrehte Brennhaare wie Fig. 2, Taf. I. Das Aussehen jüngerer Brennhaare hängt auch davon ab, ob die Seitenzellen oben oder unten die größte Breite haben (Fig. 3 und 4, Taf. I). Öfters treten im Verlaufe der Seitenzellen Querwände auf, welche die normale Gestalt des Brennhaares nicht verändern. So sah ich an einem ausgewachsenen Brennhaar jede Seitenzelle in drei gleich lange Teile geteilt. Fig. 1 der Taf. I zeigt links unten eine solche Querwand. Oft sind solche Querwände die Ursache von Mißbildungen (Fig. 7, Taf. I). Eine interessante Mißbildung anderer Art ist in Fig. 8, Taf. I, abgebildet. Es ist dies ein vierseitiges 
Brennhaar mit zwei wohlausgebildeten Zentralzellen. Diese Bildung kam dadurch zustande, daß die Zentralzellen entweder in unmittelbarer Nachbarschaft angelegt wurden oder dadurch, daß sich eine Anlage ausnahmsiveise geteilt hat. Die beiden Zentralzellen sind dann an derselben Stelle der Epidermis hindurchgedrungen und haben sich vier Epidermiszellen für das gemeinsame Postament mit emporgenommen.

Die im Vorigen gegebenen Details beziehen sich sämtlich auf Dalechampia Roezliana Mü1l. Arg. Für Tragia habe ich die Entwicklungsgeschichte nicht genauer untersucht; der vollkommen mit den Dalechampien übereinstimmende Bau der Brennhaare und das Vorkommen "großer" und "kleiner " Brennhaare bei Tragia berechtigt uns, auch für diese Gattung der Acalypheen die gleiche Entstehung der Brennhaare anzunehmen.

Wie Rittershausen nachgewiesen hat, liegen in der Epidermis der Acalypheen-Blätter sehr oft Idioblaste, welche sich durch verschiedenartig ausgebildete Kristalldrusen, oft auch nebenbei durch eine von den Epidermiszellen verschiedene Form und Größe auszeichnen. Schon Rittershausen hat an verschiedenen Stellen seiner Arbeit auf einen phylogenetischen Zusammenhang dieser in der Epidermis liegenden Kristallzellen und der Brennhaare hingewiesen. Doch wird die Phylogenie dieser Gebilde erst durch die soeben dargelegte Entwicklungsgeschichte verständlich.

Sehr wichtig ist es, daß in der Blattepidermis von Dalechampia Roezliana Müll. Arg. ebenfalls Kristalldrusenzellen vorkommen. Ich konnte nachweisen, daß diese Zellen keine echten Epidermiszellen sind, sondern daß es sich auch hier um subepidermal entstandene Gebilde handelt. Fig. 16 zeigt eine solche in der Epidermis gelegene Zelle, welche ihren Ursprung kaum mehr erkennen läßt. Sie enthält eine nach allen Richtungen gleichmäßig ausgebildete Kalkoxalatdruse, welche von einer enganliegenden Zellulosehülle umgeben ist. Bei der in Fig. 15 dargestellten Zelle, deren subepidermale Entstehung man noch ganz deutlich erkennt, ist die Kristalldruse durch Salzsäure gelöst worden, so daß die Zellulosehülle nunmehr als unregelmäßig geformter Liörper im Zellumen sichtbar ist. 
Von den zahlreichen subepidermal liegenden Drusenzellen, welche ich bei Dalechampia beobachtete, scheinen nur sehr wenige in die Epidermis emporzudringen. Doch besitzen diese Drusenzellen sehr häufig nach oben zu eine keilförmig verschmälerte Partie, welche sich, wie Fig. 14 zeigt, mehr oder weniger weit zwischen die darüberliegenden Epidermiszellen einzwängt. Zwischen den subepidermal gelegenen und den epidermal gelegenen Drusenzellen finden sich in den Präparaten alle Übergänge.

Von Dalechampia scandens $\beta$ fallax Müll. Arg. erwähnt Rittershausen, daß neben Drusen »in den Epidermiszellen « auch große prismatische Einzelkristalle mit Neigung zur Zwillingsbildung vorkommen. Es ist klar, daß auch diese Kristallzellen wie wohl die meisten oder alle anderen Drusenzellen der Acalypheen-Blätter subepidermalen Ursprungs sind, wenn sie zwischen den Epidermiszellen liegen. Einen wichtigen Anhaltspunkt dafür bieten die von Rittershausen an vielen Stellen seiner Arbeit gemachten Bemerkungen, daß die in der Epidermis gelegenen Drusenzellen stets sehr weit ins Blattinnere hinabreichen. Dasselbe zeigt ein von Rittershausen gezeichneter Blattquerschnitt von Claoxylon. Die von demselben Autor entdeckten sternförmigen "Drusenhaare " der Blätter von Plukenetia (und Fragariopsis) sind, so merkwürdig es auch auf den ersten Blick erscheinen mag, jedenfalls auch subepidermalen Ursprungs. ${ }^{1}$

1 Nach Rittershausen bestehen diese "Drusenhaare « aus papillös entwickelten Epidermiszellen, welche in ihrem Lumen eine dasselbe erfüllende Kristalldruse aus Kalziumoxalat enthalten, deren (3 bis 6) spitze Strahlen nach außen gerichtet sind und derart von der sehr dünnen Außenwand der papillösen Epidermiszelle eng umschlossen werden, daß das ganze Trichomgebilde ein sternhaarartiges Aussehen besitzt. Unter dieser Epidermiszelle liegt in der Regel eine ziemlich schmale lange Zelle, die tief in das Palisadengewebe eindringt. \& Rittershausen hält diese Gebilde für eine Modifikatio:ı der kristallführenden Brennhaare. - Ich glaube, daß Rittershausen bei der Betrachtung dieser $\gg$ Drusenhaare « denselben Fehler begangen hat wie bei der Untersuchung der Brennhaare. Die »papillöse Epidermiszelle und die unter ihr liegende »schmale, lange Zelle « entsprechen zusammen der Zentralzelle der Brennhaare, so daß auch hier die Zellulosehülle der Kristalldruse eine Zellwand vorgetäuscht haben mußte. Natürlich konnte das mächtige Längenwachstum 
Mit Benützung der von Rittershausen angegebenen Details läßt sich die Umbildung der subepidermalen Drusenzellen zu typischen Brennhaaren von Tragia etc. in der folgenden phylogenetischen Reihe übersichtlich zum Ausdruck bringen.

I. Subepidermal entstandene Drusenzellen dringen zwischen die Epidermiszellen ein, ohne daß sich die Gestalt der ersteren und die gleichmäßige Ausbildung der Kristalldrusen viel verändert: Dalechampia etc.

II. Die in die Epidermis vorgedrungenen Drusenzellen zeigen bereits eine ungleichmäßige Ausbildung der Kristalldrusen, indem sich die der Blattaußenseite zugewendeten Kristallindividuen stärker ausbilden. Die von der dünnen Außenwand der Zelle überzogenen längeren Kristalle ragen etwas über die Epidermisaußenfläche empor: Caperonia, Argyrothammia.

III. Die subepidermal entstandenen Zellen dringen durch die Epidermis und bilden nun Kristalldrusen, deren $\mathrm{nach}$ außen gewendete Kristalle ganz besonders großund lang werden. Die Außenwand der Drusenzelle ist sehr dünn, kann jedoch aus lokalmechanischen Gründen an einer bestimmten Stelle verdickt sein.

a) Die Druse zeigt 3 bis 6 mächtige Spießkristalle entwickelt; die Außenwand ist der Zellulosehülle der Druse eng anliegend und wahrscheinlich mit ihr vielfach oder ganz verwachsen: "Drusenhaare" von Plukenetia (und Fragariopsis)

oder:

b) Die Druse zeigt nur einen Kristall besonders stark ausgebildet. Die dünne Außenwand der Zelle berührt den Spießkristall nur an der Spitze und ist hier etwas verdickt; ferner ist sie hier und im unteren Teil der Kristalldruse mit der Zellulosehülle der letzteren verbunden.

der Kristallindividuen erst dann erfolgen, nachdem die Epidermis von der Drusenzelle vollkommen durchwachsen war. (Vergl. Rittershausen, Fig. 4 bis 6 und Solereder Fig. 180, R. S.) 
Durch starkes Längenwachstum der Drusenzelle und der benachbarten Epidermiszellen erhebt sich das ganze Gebilde weit über die Oberfläche des Blattes: Brennhaare von Dalechampia, Tragia etc.

Zum Schlusse komme ich der angenehmen Pflicht nach, Herrn Prof. G. Haberlandt, unter dessen Leitung vorliegende Arbeit ausgeführt wurde, sowie Herrn Prof. E. Palla für die mannigfaltige Unterstützung und Anregung, die mir von ihnen während meiner Untersuchungen zukam, den besten Dank auszusprechen. Auch Herrn Prof. R. v. Wettstein in Wien fühle ich mich für die überaus bereitwillige Überlassung des nötigen Herbarmaterials zu großem Danke verpflichtet.

\section{Literatur.}

1. Crüger Herm., Westindische Fragmente (Beschluß). Botanische Zeitung, 13. Jahrg. 1855, Nr. 36, S. 618 f.

2. Koh1 F. G., Anat. phys. Untersuchung der Kalksalze und Kieselsäure in der Pflanze. S. 164. Marburg 1889.

3. Weiß Ad., Die Pflanzenhaare. In "Karsten's Bot. Untersuchungen «, Band I. S. 464. Berlin 1867.

4. Rittershausen P., Anat. System. Untersuchung von Blatt und Axe der Acalypheen. Dissertation d. Univ. Erlangen. München 1892.

5. Solereder H., Systematische Anatomie der Dikotyledonen. S. 851 f. Stuttgart 1899.

6. Haberlandt G., Zur Anatomie und Physiologie der pflanzlichen Brennhaare. Sitzb. d. kais. Akad. d. Wiss. (math. naturw. K1.) Bd. XCIII. Wien 1886.

7. Haberlandt G., Physiologische Pflanzenanatomie, 3. Aufl. Leipzig 1904.

8. Müller Joh. (argoviensis), Acalypheae in De Candolle, Prodromus systematis naturalis regni vegetabilis, pars XV, sectio posterior, Fasc. II, p. 927.

9. Rothert W., Die Kristallzellen der Pontederiaceen. Botan. Zeitung. 58. Jahrg. 1900.

10. Gutten berg H. v., Zur Entwicklungsgeschichte der Kristallzellen im Blatte von Citrus. Sitzb. d. kais. Akad. d. Wiss. (math. naturw. K1.) Bd. CXI. Wien 1902.

11. Lewin L., Über die toxikologische Stellung der Rhaphiden. Ber. d. Deutschen Bot. Gesellschaft, Bd. 18. 1900. 


\section{Tafelerklärung.}

Tafel I (Bau und Funktion der Brennhaare).

Fig. 1 bis 4. Brennhaarformen in der Außenansicht, 1 und 2 erwachsene Brennhaare.

Fig. 5. Optischer Längschnitt durch ein ausgewachsenes Brennhaar; $S=$ Seitenzelle, $C=$ Zentralzelle.

Fig. 6. Optischer Querschnitt durch ein ausgewachsenes Brennhaar, $a$ in der Höhe der Kristalldruse, $b$ nahe an der Basis.

Fig. 7. Brennhaarabnormität.

Fig. 8. Vierseitiges Brennhaar mit zwei Zentralzellen (Abnormität).

Fig. 9. Oberes Ende der Zentralzelle, zeigt den Zellkern derselben.

Fig. 10. Oberer Teil des Brennhaares im opt. Längsschnitt.

Fig. 11 und 12. Verankerung der Kristalldruse (opt. Längschnitt).

Fig. 13. Ansicht der Verankerung von außen.

Fig. 14. Beschaffenheit der Brennhaarspitze; Ansicht derselben von drei Seiten (opt. Längschnitt), $b$ in Profilansicht.

Fig. $15 a$ bis $d$. Ausbildung der Kristalldruse an der Basis des Spießkristalls. Fig. 16. Kristallapparat mit zwei Spießkristallen.

Fig. 17. Kristallapparat; $s=$ Seitenfläche, $e=$ schräge Endfläche.

Fig. 18 und 19. Geöffnete Brennhaarspitzen.

Die Figuren 1 bis 9,15 und 16 beziehen sich auf Dalechampia Roezliana, Fig. 10 bis 14 auf Tragia Miqueliana, Fig. 17 bis 19 auf Tragia volubilis.

Tafel II (Entwicklungsgeschichte und Phylogenie).

Fig. 1 bis 7. Entwicklungsstadien im Längschnitt (Erklärung im Text).

Fig. 8 a. Epidermisaußenansicht mit jungen Brennhaarstadien.

$8 b$ ist die Vergrößerung von $8 a \beta$.

Fig. 9. Entwicklung einer $»$ Zweischneidigen Zentralzelle, Flächenansicht der Epidermis.

Fig 10. Entwicklung einer dreischneidigen «Zentralzelle, Flächenansicht der Epidermis.

Fig. 11. "Zweischneidige , die Epidermis durchdringende Zentralzelle (von der Seite gesehen).

Fig. 12. "Zweischneidige«, die Epidermis durchdringende Zentralzelle (von oben gesehen).

Fig. 13. „Dreischneidige « Zentralzelle, schief von der Seite gesehen.

Fig. 14 bis 16. Zellen mit allseitig ausgebildeten Kristalldrusen; in 14 noch unter der Epidermis liegend, in 15 und 16 bereits in die Epidermis vorgedrungen.

Alle Figuren beziehen sich auf Dalechampia Roezliana. 



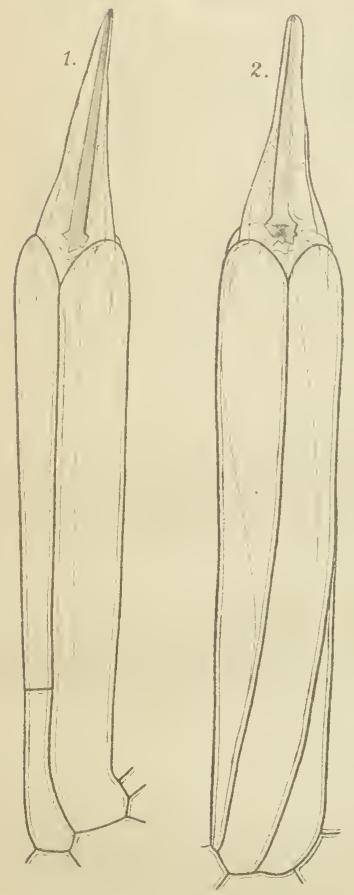

ba
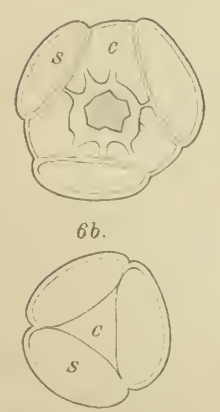

Lind: :
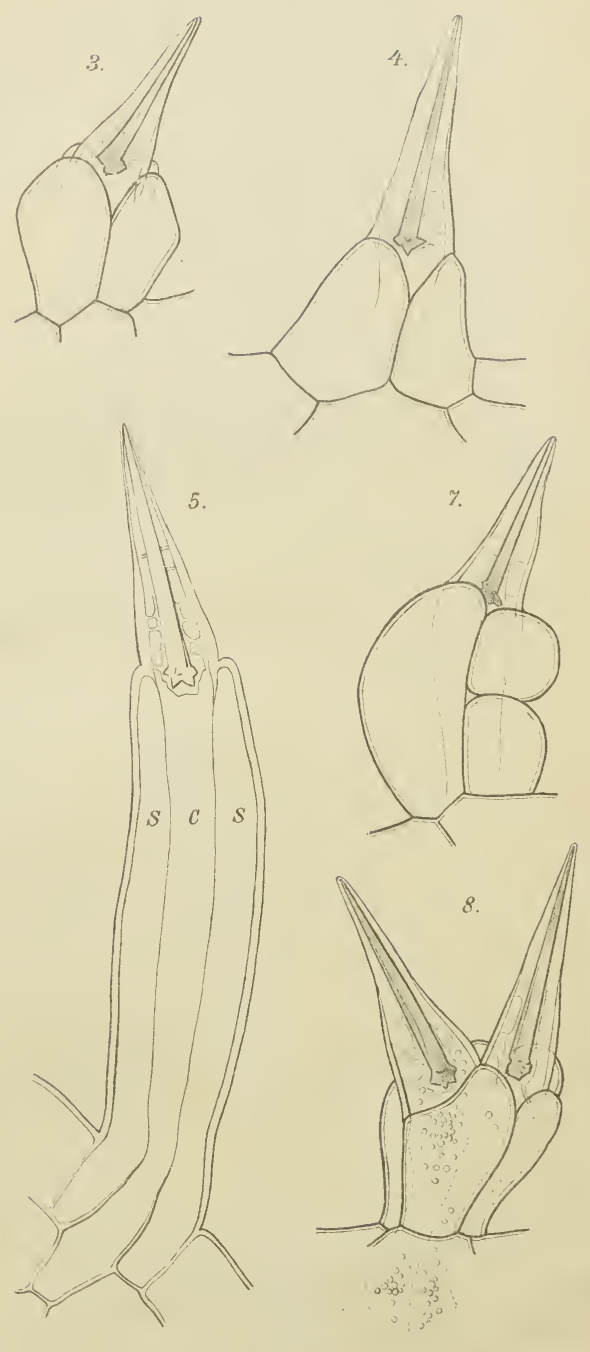
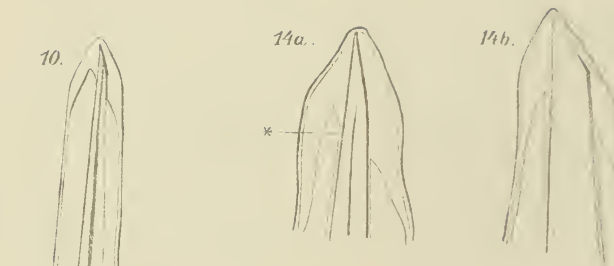

11.

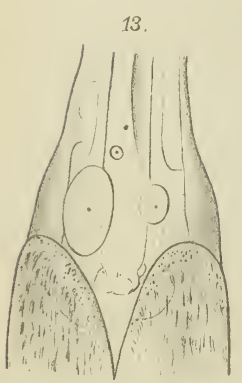

$15 \alpha$.

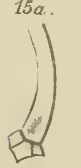

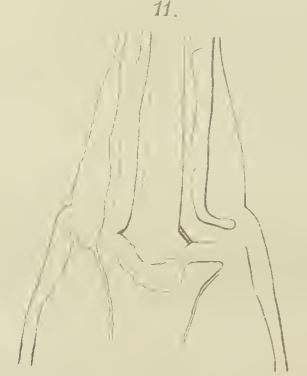
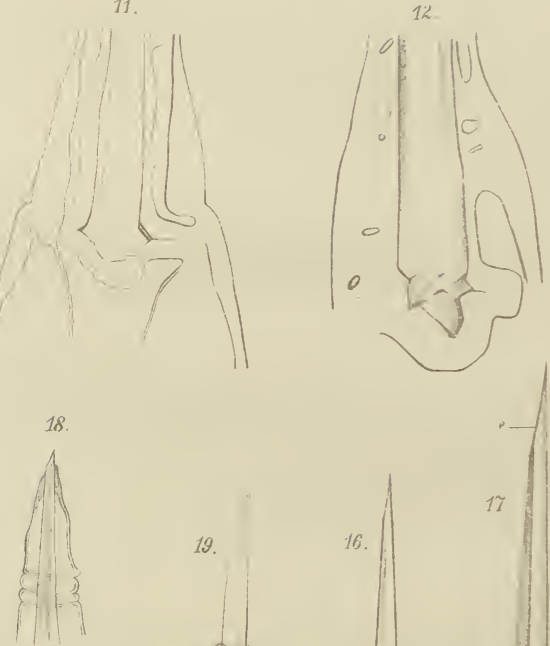

156

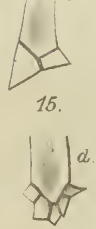

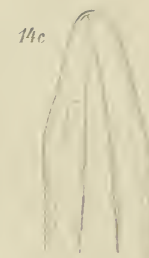

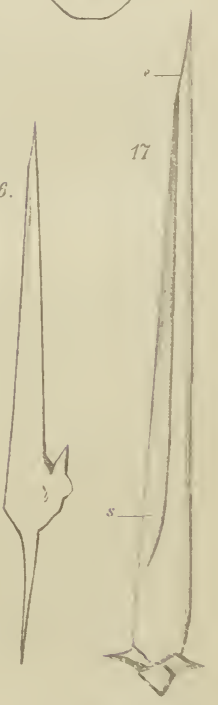

Sitzungsberichte d.kais. Akad. d. Wiss., math-naturw. Klasse, Bd.CXTV. Abt.1.1905. 



